\title{
TransPrise: a novel machine learning approach for eukaryotic promoter prediction
}

\author{
Stepan Pachganov ${ }^{\text {Equal first author, } 1}$, Khalimat Murtazalieva ${ }^{\text {Equal first author, } 2,3}{ }^{3}$, Alexei Zarubin ${ }^{4}$, Dmitry Sokolov ${ }^{5}$, Duane R \\ Chartier $^{6}$, Tatiana V Tatarinova ${ }^{\text {Corresp. } 2,7,8,9}$ \\ 1 Ugra Research Institute of Information Technologies, Khanty-Mansiysk, Russia \\ ${ }^{2}$ Vavilov Institute for General Genetics, Moscow, Russia \\ 3 Institute of bioinformatics, Moscow, Russia \\ ${ }^{4}$ Tomsk National Research Medical Center of the Russian Academy of Sciences, Research Institute of Medical Genetics, Tomsk, Russia \\ 5 Neirika Solutions, Sarov, Russia \\ 6 International Center for Art Intelligence, Inc, Los Angeles, California, United States \\ 7 Department of Biology, University of La Verne, La Verne, California, United States \\ 8 A. A. Kharkevich Institute for Information Transmission Problems, Russian Academy of Sciences, Moscow, Russia \\ ${ }^{9}$ Siberian Federal University, Krasnoyarsk, Russia \\ Corresponding Author: Tatiana V Tatarinova \\ Email address: ttatarinova@laverne.edu
}

As interest in genetic resequencing increases, so does the need for effective mathematical, computational, and statistical approaches. One of the difficult problems in genome annotation is determination of precise positions of transcription start sites. In this paper we present TransPrise - an efficient deep learning tool for prediction of positions of eukaryotic transcription start sites. Our pipeline consists of two parts: the binary classifier operates the first, and if a sequence is classified as TSS-containing the regression step follows, where the precise location of TSS is being identified. TransPrise offers significant improvement over existing promoter-prediction methods. To illustrate this, we compared predictions of TransPrise classification and regression models with the TSSPlant approach for well annotated genome of Oryza sativa. Using a computer equipped with a graphics processing unit, the run time of TransPrise is 250 minutes on a genome of $374 \mathrm{Mb}$ long. The Matthews correlation coefficient value for TransPrise is 0.79 , more than two times larger than the 0.31 for TSSPlant classification models. This represents a high level of prediction accuracy. Additionally, the mean absolute error for the regression model is $29.19 \mathrm{nt}$, allowing for accurate prediction of TSS location. TransPrise was also tested in $H$. sapiens, where mean absolute error of the regression model was $47.986 \mathrm{nt}$. We provide the full basis for the comparison and encourage users to freely access a set of our computational tools to facilitate and streamline their own analyses. The ready-to-use Docker image with all necessary packages, models, code as well as the source code of the Transprise alororithm are available at ( $h+t p$ ://compubioverne.group/). The source code is 
ready to use and customizable to predict TSS in any eukaryotic organism. 
3 Stepan Pachganov ${ }^{1}$, Khalimat Murtazalieva ${ }^{2,3}$, Alexei Zarubin ${ }^{4}$, Dmitry Sokolov ${ }^{5}$, Duane

4 Chartier $^{6}$, Tatiana V. Tatarinova ${ }^{2,7-9^{*}}$

51 Ugra Research Institute of Information Technologies, Khanty-Mansiysk, Russia

62 Vavilov Institute of General Genetics, Moscow, Russia

73 Institute of Bioinformatics, Moscow, Russia

84 Tomsk National Research Medical Center of the Russian Academy of Sciences, Research

9 Institute of Medical Genetics, Tomsk, Russia

105 Neirika Solutions, Sarov, Russia

116 International Center for Art Intelligence, Inc. Los Angeles, CA, USA

127 Department of Biology, University of La Verne, La Verne, CA, USA

138 A. A. Kharkevich Institute for Information Transmission Problems, Russian Academy of

14 Sciences, Moscow, Russia

159 Siberian Federal University, Krasnoyarsk, Russia.

16

*Corresponding author: ttatarinova@laverne.edu

\section{Abstract}

As interest in genetic resequencing increases, so does the need for effective mathematical, computational, and statistical approaches. One of the difficult problems in genome annotation is determination of precise positions of transcription start sites. In this paper we present TransPrise an efficient deep learning tool for prediction of positions of eukaryotic transcription start sites. Our pipeline consists of two parts: the binary classifier operates the first, and if a sequence is classified as TSS-containing the regression step follows, where the precise location of TSS is being identified. TransPrise offers significant improvement over existing promoter-prediction methods. To illustrate this, we compared predictions of TransPrise classification and regression models with the TSSPlant approach for well annotated genome of Oryza sativa. Using a computer equipped with a graphics processing unit, the run time of TransPrise is 250 minutes on a genome of $374 \mathrm{Mb}$ long. The Matthews correlation coefficient value for TransPrise is 0.79 , more than two times larger than the 0.31 for TSSPlant classification models. This represents a high level of prediction accuracy. Additionally, the mean absolute error for the regression model is $29.19 \mathrm{nt}$, allowing for accurate prediction of TSS location.

TransPrise was tested in $H$. sapiens, with mean absolute error of the regression model of $47.986 \mathrm{nt}$. We provide the full basis for the comparison and encourage users to freely access a set of our computational tools to facilitate and streamline their own analyses. The ready-to-use Docker image with all necessary packages, models, code as well as the source code of the 
38

39

40

41

42

43

44

45

46

47

48

49

50

51

52

53

54

55

56

57

58

59

60

61

62

63

64

65

66

67

68

69

70

71

72

73

74

75

76

77

TransPrise algorithm are available at (http://compubioverne.group/). The source code is ready to use and customizable to predict TSS in any eukaryotic organism.

\section{Introduction}

The heightened interest and lowered costs of sequencing has led to intensified use of mathematical and statistical tools to improve the accuracy and replicability of genomic analysis. Until recently, there has been a relatively simplistic and deterministic notion of promoter organization and the creation of functional models of gene regulation. We are at a turning point in genetic research we are actively assembling and refining the "dictionary" of genetics, but we are in the infancy of development of "genetic grammar". Multiple questions remain to be addressed: what do the individual "words" mean? How does genotype translate to phenotype? This is very much like the time in physics, over 100 years ago, when the classical approach had to be replaced by quantum theory and statistical mechanics. We cannot understand genetics without powerful statistical and computational tools for analysis and for verifying our hypotheses regarding genome manifestations.

Thousands of eukaryotic genomes have been sequenced so far (https://www.ncbi.nlm.nih.gov/genome/browse/\#!/eukaryotes/), including animals $(1,590)$, fungi $(3,275)$, and plants $(665)$. As of 2018, these genomes are at various assembly levels, with 840 genomes assembled at the level of chromosomes, 46 are complete, 1,191 are in contigs, and 4,057 are at the level of genomic scaffolds. Genomic projects are not limited to sequencing and genome assembly. Re-sequencing large populations is becoming an important tool to unravel population structure, detect signatures of selection and to map quantitative trait loci (QTL) (Atwell et al. 2010). As resequencing costs plummet and technology platforms continue to expand throughput (e.g. Illumina NovoSeq), genomics communities are now contemplating the possibilities of resequencing entire germplasm collections to detect the vast majority of existing alleles and haplotypes. One essential requirement to capture allelic diversity is to have high-quality reference genomes that span the breadth of genomic diversity for mapping resequencing data.

Understanding the functional role of a given single-nucleotide polymorphism or a structural variant requires knowledge of its location with respect to coding and regulatory regions and the elements involved ( $\mathrm{Li}$ et al. 2015; Mulder 2018; Tatarinova et al. 2016; Triska et al. 2017b). In addition, the regulatory role of a transcription factor binding site (TBFS) has been demonstrated to depend on the position of the TFBS with respect to the transcription start site (TSS) (Berendzen et al. 2006; Pritsker et al. 2004). Determination of the precise location of TSS is an essential preparatory step for motif discovery and reconstruction of gene regulatory networks (Triska et al. 2017a; Troukhan et al. 2009). The interaction of a vast number of proteins, multi-subunit complexes, and DNA binding sites make eukaryotic transcriptional regulation an extremely convoluted process (Eckardt 2014). Therefore, it is vitally important to have reliable methods for 
78 promoter prediction and analysis of regulatory elements if we are to enhance our capacity to 79 engineer crops or to select therapeutic targets.

80

81

82

83

84

85

86

87

88

89

90

91

92

93

94

95

96

97

98

99

100

101

102

103

104

105

106

107

108

109

110

111

112

113

114

115

116

117

Homology-based prediction of coding regions is a relatively straightforward procedure (Keilwagen et al. 2018). Multiple tools and pipelines exist for finding positions and functions of genes, such as MAKER (Campbell et al. 2014a; Campbell et al. 2014b; Holt \& Yandell 2011), BREAKER (Hoff et al. 2015), Augustus (Stanke \& Morgenstern 2005), GeneMarkHMM (Lukashin 1998), FgeneSH (Salamov \& Solovyev 2000), and many others. These pipelines achieve remarkably high accuracy in homology-based gene finding; however, homology between species does not necessarily extend beyond coding regions, and, therefore, accurate prediction of promoters is difficult. It has been reported that even state-of-the art modern methods of promoter mapping are incapable of achieving 100\% accuracy (Alexandrov et al. 2009; Alexandrov et al. 2006; Batut et al. 2013; Carninci et al. 2006; Herbig et al. 2013; Kawaji et al. 2006; Kawaji et al. 2014; Morton et al. 2014; Tatarinova et al. 2013b; Tatarinova et al. 2016; Troukhan et al. 2009). For example, current annotations of rice (MSU7) and maize (B73, 6a) contain 56K and 63K predicted genes, correspondingly (Liseron-Monfils et al. 2013), and for nearly two thirds of those genes, TSS is not identified precisely. Traditional deterministic approaches can predict only $\sim 50 \%$ of promoters with one false positive promoter predicted every $700-1000 \mathrm{nt}$ of the genome (Shahmuradov \& Solovyev 2015; Solovyev et al. 2010). This accuracy is insufficient to make reliable predictions, because we expect one promoter per 10,000-20,000 nt of a genome. PromH (Solovyev \& Shahmuradov 2003) used conservation of promoter functional components between orthologous genes to improve prediction of TSS. PromH was able to predict TSS within $10 \mathrm{nt}$ for $90 \%$ of the TATA+ promoters and for $40 \%$ of TATA- genes, but only if there are highly similar homologous sequences from closely related species. The TSSer algorithm (Troukhan et al. 2009) that combined positional frequency of 5' EST/RNA-Seq matches on genomic DNA with gene models was able to accurately predict one transcription start site per locus. However, it is now accepted that alternative promoters are associated with differential expression in various tissues and chromatin states (Rye et al. 2014). A nonparametric maximum likelihood approach, NPEST (Tatarinova et al. 2013b), can predict multiple TSSs per locus if 5' EST/CAGE/mRNA data are available. Promoter sequences predicted by NPEST were demonstrated to be more accurate for the $A$. thaliana genome than sequences identified in several gold standard databases, such as TAIR, Plant Prom DB and Plant Promoter Database. However, it is difficult to identify TSS from RNA-Seq alone, since only $26 \%$ of genes display a maximum of the RNA-Seq coverage in the range [TSS-50, TSS +250], and only $60 \%$ of genes display this maximum in the range [TSS-50, TSS+550] (Steijger et al. 2013). Enough RNA-Seq and CAGE data is not available for all genomes of interest. Therefore, it is imperative to develop alternative strategies.

There are several factors complicating the process of TSS prediction, such as existence of multiple TSS per locus. Studies on mammalian and plant genomes have revealed that many eukaryotic genes are associated with multiple distinct promoters (Batut et al. 2013; Farrell \& Bassett 2007;

Peer) reviewing PDF | (2019:06:38847:1:3:NEW 25 Sep 2019) 
118 Louzada 2007; Morton et al. 2014; Tatarinova et al. 2013b). Moreover, eukaryotic promoters are 119 characterized by multiple TSSs and can be classified based on the distribution and utilization of 120 their collective TSSs. Consequently, the association with several distinct promoters allows for a

121

122

123

124

125

126

127

128

129

130

131

132

133

134

135

136

137

138

139

140

141

142

143

144

145

146

147

148

149

150

151

152

153

154

155

156

157

single gene to encode various protein isoforms (Sandelin et al. 2007).

In addition, the performance of standard promoter identification in grasses and warm-blooded vertebrates is complicated by the existence of two classes of genes in those organisms: $\mathrm{GC}_{3}-$ rich and -poor ones (where $\mathrm{GC}_{3}$ is the fraction of $\mathrm{Cs}$ and $\mathrm{Gs}$ in the third position of codons). Nucleotide composition of $\mathrm{GC}_{3}$-rich genes differs from $\mathrm{GC}_{3}$-poor ones; they also have higher variability of gene expression levels (resulting in fewer full-length mRNA support) (Elhaik et al. 2014; Elhaik \& Tatarinova 2012; Tatarinova et al. 2013a). Since a majority of the stress-related and tissuespecific genes are $\mathrm{GC}_{3}$-rich (Chan et al. 2017b), refinement of promoter prediction pipeline is an essential task.

Many genomic features are associated with the location of promoter: positional frequency of $5^{\prime}$ ESTs and RNA-Seq matches on genomic DNA, nucleotide distribution, DNA methylation, distribution of SNPs, characteristic regulatory elements. Incorporation of those data types allows accurate prediction of TSS. A recently developed tool, TSSPlant (Shahmuradov et al. 2017), based on the Expectation Maximization (EM) algorithm, achieves significantly higher accuracy compared to state-of-the art promoter prediction programs for both TATA-containing and TATAless promoters. Umarov \& Solovyev (2017) developed a deep learning a deep learning approach to characterize genomic regions as promoters and non-promoters; and Triska et al. (2017b) applied it to rice genome, achieving 99\% accuracy in classification of $250 \mathrm{nt}$ long regions. However, the question of the specific location of the TSS within these $250 \mathrm{nt}$ long windows remains open.

This paper presents a novel, accurate, and data-type independent procedure for TSS prediction that can incorporate multiple data types. Our method is based on machine learning approach that is capable of uncovering intricate properties of promoter regions and achieving much higher accuracy than deterministic methods (Umarov \& Solovyev 2017). Our novel method aims to identify the position of the start of transcription with the highest possible precision using nucleotide composition alone. The method can predict multiple transcription start sites per locus. It is data-type agnostic and can be extended to incorporate additional biological features. We present a set of computational tools, a user-friendly public interface and a curated online database to facilitate these analyses.

\section{Materials and Methods}

\section{Selection of genome annotation version}

We selected rice chromosomes and Genome Annotation release 7 (MSUv7, http://rice.plantbiology.msu.edu). There are two commonly used annotations of rice: MSU (Kawahara et al. 2013) and FgeneSH (Zhang et al. 2008). The Fgenesh gene prediction set contains 
158

159

160

161

162

163

164

165

166

167

168

169

170

171

172

173

174

175

176

177

178

179

180

181

182

183

184

185

186

187

188

189

190

191

192

193

194

195

18,389 high quality ( $5^{\prime}$ full, with mRNA support) gene models, while the MSU gene prediction set contains 20,367 high quality gene models (Tatarinova et al. 2016). We used Fgenesh mRNAbased gene prediction models, since Fgenesh-annotated promoters have a more pronounced nucleotide consensus as compared to the promoters annotated by MSU (Triska et al. 2017b). Fgenesh was successfully used to annotate several plant genomes (Chan et al. 2017a; Chan et al. 2017b; Davis et al. 2010; Ito et al. 2005; Jiang et al. 2015; Nasiri et al. 2013; Sanusi et al. 2018; Sheshadri et al. 2018; Yao et al. 2005). Therefore, we selected the Fgenesh annotation as the gold standard for our analysis. To obtain the highest quality dataset, pseudogenes, transposable elements, and genes with 5' UTR shorter than $20 \mathrm{nt}$ or longer than $1000 \mathrm{nt}$ have been excluded.

\section{Training, validation and test sets}

The procedure consists of two steps: classification (dividing the genome into "promoters" and "non-promoters") and regression (finding the position of TSS inside the sequence identified as "promoter"). The genomic sequence of Oryza sativa had been divided into the testing and training sets. The training set contains three files:

1. Training “non-promoter" dataset contains sequences extracted from random genomic positions separated from experimentally validated transcription start sites by $2000 \mathrm{nt}$. This dataset contains mostly intergenic regions. All sequences are $2000 \mathrm{nt}$ long.

2. Training "promoter" dataset contains sequences [TSS-1000; TSS+999] from the all chromosomes with length $2000 \mathrm{nt}$.

3. File with indicators of TSS positions, containing $(2000 \times 1)$ matrices that correspond to positions of biologically validated TSS in every training sequence ("1" TSS, " 0 " not TSS position).

The same set of files was created for the testing dataset. Since the procedure has multiple steps (classification and regression), training and testing sets were selected at each step of the method.

The following procedure was used to assemble the dataset for the classification model:

1) "Non-promoters": $1 / 4$ of the examples chosen from the training "non-promoter" dataset, randomly selecting 512 long sequences from $2000 \mathrm{nt}$ long regions.

2) "Promoters sans TSS": $1 / 4$ of the examples were randomly selected from the training "promoter" dataset, making sure that the chosen $512 \mathrm{nt}$ long fragment did not overlap the region [TSS-50, TSS+50].

3) "TSS vicinity": $1 / 2$ of the examples extracted from the training "promoter" dataset, containing only one TSS in a random position within the $512 \mathrm{nt}$ long sequence, with a restriction that it should be in the $[250,450]$ fragment.

The dataset for the regression model was assembled using sequences that contain one validated TSSs in a randomly selected position of the $[250,450]$ fragment. The datasets are represented as (512×4) nucleotide matrices $M$ with 512 columns and 4 rows. The $1^{\text {st }}$ row contains delta function 
$196 \delta\left(\mathrm{x}_{\mathrm{i}}=\mathrm{A}\right)$ - it is equal to 1 if there is nucleotide " $\mathrm{A}$ " in the $\mathrm{i}^{\text {th }}$ position of the sequence and 0 otherwise. 197 Similarly, $2^{\text {nd }}, 3^{\text {rd }}$, and $4^{\text {th }}$ rows correspond to nucleotides $C, G$ and $T$.

198

199

200 We implemented the Convolutional Neural Networks (CNN) using the Keras library for training 201 (https://keras.io/).

202

203

Classification and Regression models training

204 The dataset for the classification contains equal numbers of positive and negative examples. The 205 matrices $(512 \times 4)$ described above are input into the model. The CNN architecture (Figure 1) 206 started with four parallel convolutional layers (composed of 128 filters with 2, 4, 8 and 16 kernel 207 sizes) ReLU, was used as activation function followed by concatenation. After concatenation layer 208 we used convolution, batch normalization, max pooling layers twice. The First convolution had 209128 filters and the second had 16. There were one kernel size and ReLU activation in both 210 situations. To help regularize the model, we used the 0.5 Dropout technique. The signal is fed to 211 two fully connected layers with ReLU activation functions consisted of 256 and 128 neurons, 212 followed by batch normalization. The output layer had a sigmoid activation function.

213

214 Whole Genome Sequencing (WGS) processing

215 TransPrise also works with WGS data in fasta format. We prepared a Python script that with 250 216 minutes run time on a genome of $374 \mathrm{Mb}$ long. It uses a sliding window for extraction of $512 \mathrm{nt}$ 217 long sequences with the step size 4 nt. If classification step identified a fragment as TSS218 containing, this fragment will be passed to the regression step. Prediction vector is compared to 219 the TSS model using L1 norm. The default value for the similarity threshold is 600 , but it can be 220 modified by users. The only restriction of our algorithm is that alternative TSSs must be more than $221100 \mathrm{nt}$ of each other.

222 


\section{Model evaluation}

224 For the purpose of the K-fold cross-validation, the dataset is randomly divided into K equal-size 225 subsets. Of the K subsets, a single subset is retained as the validation data for testing the model, 226 and the remaining (K-1) subsets are used for training. The cross-validation process is then repeated 227 K times (the "folds"), with each of the K subsamples used exactly once as the validation data. 228 Then, the results from $\mathrm{K}$ folds are averaged. The advantage of $\mathrm{K}$-fold cross-validation is that all 229 observations are used for both training and validation, and each observation is used for validation 230 exactly once. We conducted 10-fold cross-validation (dataset was divided into training and 231 validation sets in 9:1 ratio; validation set was used to avoid overfitting and find the optimal number 232 of learning epochs). The ROC curves obtained in 10-fold cross-validation are presented in the 233 "Results" section. We determined that the optimal number of learning epochs is five. After the 234 model training, we tested our model using the test set and calculated Accuracy (Ac), Sensitivity 235 (Se), Specificity (Sp), and the Matthews Correlation Coefficient (CC):

236

237 Specificity $=\frac{T P}{T P+F P}$,

238 Sensitivity $=\frac{T P}{T P+F N}$,

239 Accuracy $=\frac{T P+T N}{T P+T N+F P+F N}$,

$240 C C=\frac{T P \times T N+F P \times F N}{\sqrt{(T P+F P)(T P+F N)(T N+F P)(T N+F N)}}$,

242 where TP - true positive, TN - true negative, FP - false positive, FN - false negative. The input of 243 the regression model has the same format as the classification model input. TSS is assumed to be 244 located at a random position between nucleotides 250 and 450. There is only one difference 245 between classification and regression models: in the output layer the activation function is replaced 246 by a linear function.

247

248 We trained the classification and regression models using the Keras Library and performed 10249 fold cross validation procedures for them. The algorithm for TSS prediction is presented in Figure 250 1. For every fold, we carried out five learning epochs. the complete learning time was 35 seconds 251 on average. We performed 10-fold cross-validation and calculated the average value of mean 252 absolute error (MAE) to estimate the accuracy of TSS position prediction, where $y_{i}$ - position of 253 TSS in test set (assumed to be accurate), and $x_{i}$ - predicted position of TSS.

254

$$
M A E=\frac{\sum_{i=1}^{n}\left|y_{i}-x_{i}\right|}{n}
$$

256 Figure 2 shows the flowchart for model building and validation. 
257

258

259

260

261

262

263

264

265

266

267

268

269

270

271

272

273

274

275

276

277

278

279

280

281

282

283

284

285

286

287

288

289

290

291

292

\section{Results}

\section{Classification}

To reduce the influence of how the data is split on the resulting testing statistics, we carried out a 10 -fold cross-validation (CV) procedure for "internal" validation and supplemented it by the "external" validation of the rice chromosome 2 (excluded from the training set). We have randomly divided the dataset into 10 partitions and used each partition as the testing data while training the model on the remaining partitions. The ROC (Receiver Operating Characteristic) curve represents dependence of sensitivity on the specificity. It is a graph showing the performance of a classification model at all classification thresholds. AUC-ROC curves for classifier obtained in 10 -fold "internal" cross-validation are presented in Figure 3. Accuracy $=0.88, \mathrm{Se}=0.84, \mathrm{Sp}=$ $0.92, \mathrm{CC}=0.79$, $\mathrm{AUC}=0.94$.

Then we have selected the best model and evaluated its performance on rice chromosome 2 ("external" validation dataset) and compared with TSSPlant. Dataset for "external" validation contained 2000 nucleotide sequences with length 512 nt, where 1000 examples - "non-TSS" sequences and 1000 - "TSS" sequences). For the "external" validation we calculated the Matthews correlation coefficient (MCC), Accuracy (Ac), Sensitivity (Se), Specificity (Sp), and Area Under the ROC Curve (AUC-ROC) for classification models. The results of the "external" validation are presented in Table 1.

We have clearly shown that the classification model (Stage 1 of the pipeline) already achieves high accuracy (0.88). Regression (Stage 2) will further refine the prediction.

\section{Regression}

Figure 4 shows the error density curves obtained in the 10-fold cross validation procedure for regression models. The mean absolute error (MAE) for regression model was 29.19 nt. The mean difference between predicted and true TSS, if the guess is random, is $66 \mathrm{nt}$.

We compared the accuracy of promoter prediction for TSSPlant and TransPrise. For this purpose, we selected rice chromosomes 1 and 2. The testing was performed for entire chromosomes, without filtering for possible TSS containing regions, located upstream of the ATG. 
293 These two chromosomes contain 5,298 high quality experimentally validated TSS, defined as 294 follows:

295 1) Locus does not correspond to transposable element

296 2) Locus has experimental support (full-length mRNA)

297 3) If multiple isoforms are predicted, the "representative" is used

298 4) Size of the $5^{\prime}$ UTR is at least $20 \mathrm{nt}$.

299

300 For these two chromosomes, TSSPlant predicted 153,009 TSSs, while TransPrise has found 301 13,765 sites. Of TSSPlant predictions, 10,721 ( 7\%) were located within $1000 \mathrm{nt}$ from validated 302 TSS. Of TransPrise predictions, 3,989 ( 29\%) were located within $1000 \mathrm{nt}$ from validated TSS. 303 Additionally, TransPrise predictions tend to be closer to validated TSS than TSSPlant predictions 304 (Figure 5).

305

306

307

308

309

310

311

312

313

314

315

316

317

318

319

320

321

322

323

324

325

326

327

328

\section{Validation of TransPrise in Homo sapiens}

We have used Cap Analysis of Gene Expression (CAGE) data from DBTSS database (https://dbtss.hgc.jp/), using the HG19 version of the human genome (Suzuki et al. 2018). The model was trained on all chromosomes except chromosome 8 and tested on chromosome 8 . Accuracy of the classification model was 0.778 , sensitivity 0.816 , specificity 0.74 , and Matthews correlation coefficient 0.57 . The mean absolute error of the regression model was $47.986 \mathrm{nt}$.

\section{Discussion}

We have developed an efficient deep learning approach for prediction of the position of transcription start sites in eukaryotes using properties of a nucleotide sequence. The approach is data-type independent and allows incorporation of additional data types (such as RNA-seq and tissue specific DNA methylation), refining positions of TSS for tissue-specific and stress-specific expression.

We compared TransPrise with the TSSPlant approach on an independent test set composed of 2000 nucleotide sequences. All sequences were 512 nt long, and 1000 sequences did not contain TSS ("non-TSS"), and 1000 contained TSS ("TSS" sequences). The Matthews correlation coefficient value for TransPrise is more than twice larger than for TSSPlant classification models (0.79 vs. 0.31), indicating the significantly higher efficiency of TransPrise in distinguishing between regions that contain and do not contain starts of transcription. Additionally, a regression model was created for precise localization of TSS within the sequence classified as a "promoter". We validated our regression model on a test set composed of 1000 "TSS" sequences selected from chromosome 2 and calculated the mean absolute error to be $47 \mathrm{nt}$. 
Another important genome annotation task is identification of functional motifs. The architecture of TransPrise is especially designed for that. The first convolution layer is composed of four different kernel size filters $\left(a_{i, j}\right)-4 \times 2,4 \times 4,4 \times 8,4 \times 16$ matrices, where $[\mathrm{i}:[1,0,0,0](\mathrm{A}),[0,1,0,0](\mathrm{T}),[0,0,1,0](\mathrm{C}),[0,0,0,1](\mathrm{G})]$ and $[\mathrm{j}: 2,4,8,16$ - length of motif sequence] (in total 128 filters of each type). After model training, the filters correspond to PWM (positionspecific weight matrix) describing informative sequences in promoters and can be visualized as sequence logos. Several filter motifs correspond to known regulatory elements: TGGGCC (Lu et al. 2013), CGATT (Chen et al. 2016), ACTCAT (Weltmeier et al. 2006), and CGCG box (Yang \& Poovaiah 2002). Motif TGGGCC is targeted by the TCP transcription factor through its interaction with proliferating cell nuclear antigens PCF1 and PCF2 (Lu et al. 2013); ACTCAT motif is a typical binding site of basic leucine zipper (bZIP) transcription factor (Weltmeier et al. 2006); CGCG cis-elements are found in promoters of stress-related genes, for example involved in ethylene signaling, abscisic acid signaling, and light signal perception. They are bound by AtSR 1 transcription factor (Yang \& Poovaiah 2002).

Figure 6 shows "filter" motifs that correspond to two well-characterized features of eukaryotic promoters: Initiator element CA and TATA-box (Smale \& Baltimore 1989; Zhu et al. 1995). Therefore, we have shown that at least some of the features selected by the model as informative for identification of TSS correspond to known, biologically validated regulatory elements, overrepresented at or near the start of transcription. We believe that other features may correspond to unknown regulatory elements.

\section{Conclusions}

TransPrise is an advanced tool for improving genome annotation. This is symbolic of a mathematical approach to biology, which is increasingly significant. As the pressure to annotate genomes increases with plummeting sequencing costs, we must focus efforts on understanding the significance of individual genomic regions. TransPrise can predict important regulatory regions and identify characteristic motifs at a high level of efficiency relative to existing approaches. TransPrise is data-type and species independent tool that can be easily installed and customized.

\section{Software availability}

We offer a simple and efficient way to deploy our training models for most users' devices without having to install third-party deep learning packages. We have implemented the ready-to-use Docker image with all necessary packages, models and code (http://compubioverne.group/). The 
368 source code for our program TransPrise is also available on GitHub 369 (https://github.com/StepanAbstro/TransPrise) and is ready to use for new model training.

370

371

372

373

374

375

376

377

378

379

380

381

382

383

384

385

386

387

388

389

390

391

392

393

394

395

396

397

398

399

400

401

402

403

404

405

406

407

408

409

410

411

412

413

414

415

416

\section{Bibliography}

Alexandrov NN, Brover VV, Freidin S, Troukhan ME, Tatarinova TV, Zhang H, Swaller TJ, Lu YP, Bouck J, Flavell RB, and Feldmann KA. 2009. Insights into corn genes derived from large-scale cDNA sequencing. Plant Mol Biol 69:179-194. 10.1007/s11103-008-9415-4

Alexandrov NN, Troukhan ME, Brover VV, Tatarinova T, Flavell RB, and Feldmann KA. 2006. Features of Arabidopsis Genes and Genome Discovered using Full-length cDNAs. Plant Mol Biol 60:69-85. 10.1007/s11103-005-2564-9

Atwell S, Huang YS, Vilhjalmsson BJ, Willems G, Horton M, Li Y, Meng D, Platt A, Tarone AM, Hu TT, Jiang R, Muliyati NW, Zhang X, Amer MA, Baxter I, Brachi B, Chory J, Dean C, Debieu M, de Meaux J, Ecker JR, Faure N, Kniskern JM, Jones JD, Michael T, Nemri A, Roux F, Salt DE, Tang C, Todesco M, Traw MB, Weigel D, Marjoram P, Borevitz JO, Bergelson J, and Nordborg M. 2010. Genome-wide association study of 107 phenotypes in Arabidopsis thaliana inbred lines. Nature 465:627-631. 10.1038/nature08800

Batut P, Dobin A, Plessy C, Carninci P, and Gingeras TR. 2013. High-fidelity promoter profiling reveals widespread alternative promoter usage and transposon-driven developmental gene expression. Genome Res 23:169-180. 10.1101/gr.139618.112

Berendzen KW, Stüber K, Harter K, and Wanke D. 2006. Cis-motifs upstream of the transcription and translation initiation sites are effectively revealed by their positional disequilibrium in eukaryote genomes using frequency distribution curves. BMC Bioinformatics 7:522. 10.1186/1471-2105-7-522

Campbell MS, Holt C, Moore B, and Yandell M. 2014a. Genome Annotation and Curation Using MAKER and MAKER-P. Current Protocols in Bioinformatics, 4.11.11-14.11.39.

Campbell MS, Law M, Holt C, Stein JC, Moghe GD, Hufnagel DE, Lei J, Achawanantakun R, Jiao D, Lawrence CJ, Ware D, Shiu SH, Childs KL, Sun Y, Jiang N, and Yandell M. 2014b. MAKER-P: a tool kit for the rapid creation, management, and quality control of plant genome annotations. Plant Physiol 164:513-524. 10.1104/pp.113.230144

Carninci P, Sandelin A, Lenhard B, Katayama S, Shimokawa K, Ponjavic J, Semple CAM, Taylor MS, Engström PG, Frith MC, Forrest ARR, Alkema WB, Tan SL, Plessy C, Kodzius R, Ravasi T, Kasukawa T, Fukuda S, Kanamori-Katayama M, Kitazume Y, Kawaji H, Kai C, Nakamura M, Konno H, Nakano K, Mottagui-Tabar S, Arner P, Chesi A, Gustincich S, Persichetti F, Suzuki H, Grimmond SM, Wells CA, Orlando V, Wahlestedt C, Liu ET, Harbers M, Kawai J, Bajic VB, Hume DA, and Hayashizaki Y. 2006. Genome-wide analysis of mammalian promoter architecture and evolution. Nat Genet 38:626-635. $10.1038 /$ ng 1789

Chan KL, Rosli R, Tatarinova TV, Hogan M, Firdaus-Raih M, and Low EL. 2017a. Seqping: gene prediction pipeline for plant genomes using self-training gene models and transcriptomic data. BMC Bioinformatics 18:1426. 10.1186/s12859-016-1426-6

Chan KL, Tatarinova TV, Rosli R, Amiruddin N, Azizi N, Halim MAA, Sanusi N, Jayanthi N, Ponomarenko P, Triska M, Solovyev V, Firdaus-Raih M, Sambanthamurthi R, Murphy D, and Low EL. 2017b. Evidence-based gene models for structural and functional annotations of the oil palm genome. Biol Direct 12:21. 10.1186/s13062-017-0191-4

Chen J, Yi Q, Cao Y, Wei B, Zheng L, Xiao Q, Xie Y, Gu Y, Li Y, Huang H, Wang Y, Hou X, Long T, Zhang J, Liu H, Liu Y, Yu G, and Huang Y. 2016. ZmbZIP91 regulates expression of starch synthesis-related genes by binding to ACTCAT elements in their promoters. J Exp Bot 67:1327-1338. 10.1093/jxb/erv527

Peer) reviewing PDF | (2019:06:38847:1:3:NEW 25 Sep 2019) 
417 Davis TM, Shields ME, Zhang Q, Tombolato-Terzić D, Bennetzen JL, Pontaroli AC, Wang H, Yao

418

419

420

421

422

423

424

425

426

427

428

429

430

431

432

433

434

435

436

437

438

439

440

441

442

443

444

445

446

447

448

449

450

451

452

453

454

455

456

457

458

459

460

461

462

463

464

465

466

$Q$, SanMiguel $P$, and Folta KM. 2010. An examination of targeted gene neighborhoods in strawberry. BMC Plant Biol 10:81. 10.1186/1471-2229-10-81

Eckardt NA. 2014. Unexpected Structure of Plant Promoters. Plant Cell 26:2726-2726. 10.1105/tpc. 114.129239

Elhaik E, Pellegrini M, and Tatarinova TV. 2014. Gene expression and nucleotide composition are associated with genic methylation level in Oryza sativa. BMC Bioinformatics 15:23. 10.1186/1471-2105-15-23

Elhaik E, and Tatarinova T. 2012. GC3 Biology in Eukaryotes and Prokaryotes.

Farrell RE, and Bassett CL. 2007. Multiple Transcript Initiation as a Mechanism for Regulating Gene Expression. Regulation of Gene Expression in Plants, 39-66.

Herbig A, Sharma C, and Nieselt K. 2013. Automated transcription start site prediction for comparative Transcriptomics using the SuperGenome. EMBnetjournal 19:19. 10.14806/ej.19.a.617

Hoff KJ, Lange S, Lomsadze A, Borodovsky M, and Stanke M. 2015. BRAKER1: Unsupervised RNA-Seq-Based Genome Annotation with GeneMark-ET and AUGUSTUS: Table 1. Bioinformatics 32:767-769. 10.1093/bioinformatics/btv661

Holt C, and Yandell M. 2011. MAKER2: an annotation pipeline and genome-database management tool for second-generation genome projects. BMC Bioinformatics 12:491. 10.1186/1471-2105-12-491

Ito Y, Arikawa K, Antonio BA, Ohta I, Naito S, Mukai Y, Shimano A, Masukawa M, Shibata M, Yamamoto M, Ito Y, Yokoyama J, Sakai Y, Sakata K, Nagamura Y, Namiki N, Matsumoto T, Higo K, and Sasaki T. 2005. Rice Annotation Database (RAD): a contig-oriented database for map-based rice genomics. Nucleic Acids Res 33:D651-655. 10.1093/nar/gki083

Jiang Y, Ding M, Cao Y, Yang F, Zhang H, He S, Dai H, Hao H, and Rong J. 2015. Genetic fine mapping and candidate gene analysis of the Gossypium hirsutum Ligon lintless-1 (Li1) mutant on chromosome 22(D). Mol Genet Genomics 290:2199-2211. 10.1007/s00438015-1070-2

Kawahara Y, de la Bastide M, Hamilton JP, Kanamori H, McCombie WR, Ouyang S, Schwartz DC, Tanaka T, Wu J, Zhou S, Childs KL, Davidson RM, Lin H, Quesada-Ocampo L, Vaillancourt B, Sakai H, Lee SS, Kim J, Numa H, Itoh T, Buell CR, and Matsumoto T. 2013. Improvement of the Oryza sativa Nipponbare reference genome using next generation sequence and optical map data. Rice 6:4. 10.1186/1939-8433-6-4

Kawaji H, Kasukawa T, Fukuda S, Katayama S, Kai C, Kawai J, Carninci P, and Hayashizaki Y. 2006. CAGE Basic/Analysis Databases: the CAGE resource for comprehensive promoter analysis. Nucleic Acids Res 34:D632-636. 10.1093/nar/gkj034

Kawaji H, Lizio M, Itoh M, Kanamori-Katayama M, Kaiho A, Nishiyori-Sueki H, Shin JW, KojimaIshiyama M, Kawano M, Murata M, Ninomiya-Fukuda N, Ishikawa-Kato S, Nagao-Sato S, Noma S, Hayashizaki Y, Forrest ARR, Carninci P, and Consortium F. 2014. Comparison of CAGE and RNA-seq transcriptome profiling using clonally amplified and singlemolecule next-generation sequencing. Genome Res 24:708-717. 10.1101/gr.156232.113

Keilwagen J, Hartung F, Paulini M, Twardziok SO, and Grau J. 2018. Combining RNA-seq data and homology-based gene prediction for plants, animals and fungi. BMC Bioinformatics 19:189. 10.1186/s12859-018-2203-5

Li MJ, Yan B, Sham PC, and Wang J. 2015. Exploring the function of genetic variants in the noncoding genomic regions: approaches for identifying human regulatory variants affecting gene expression. Brief Bioinform 16:393-412. 10.1093/bib/bbu018

Liseron-Monfils C, Lewis T, Ashlock D, McNicholas PD, Fauteux F, Strömvik M, and Raizada MN. 2013. Promzea: a pipeline for discovery of co-regulatory motifs in maize and other plant

Peer) reviewing PDF | (2019:06:38847:1:3:NEW 25 Sep 2019) 
467

468

469

470

471

472

473

474

475

476

477

478

479

480

481

482

483

484

485

486

487

488

489

490

491

492

493

494

495

496

497

498

499

500

501

502

503

504

505

506

507

508

509

510

511

512

513

514

515

516 species and its application to the anthocyanin and phlobaphene biosynthetic pathways and the Maize Development Atlas. BMC Plant Biol 13:42. 10.1186/1471-2229-13-42

Louzada ES. 2007. Alternative Processing as a Mechanism for Regulating Gene Expression. Regulation of Gene Expression in Plants, 67-100.

Lu Z, Yu H, Xiong G, Wang J, Jiao Y, Liu G, Jing Y, Meng X, Hu X, Qian Q, Fu X, Wang Y, and $\mathrm{Li}$ J. 2013. Genome-wide binding analysis of the transcription activator ideal plant architecture 1 reveals a complex network regulating rice plant architecture. Plant Cell 25:3743-3759. 10.1105/tpc.113.113639

Lukashin A. 1998. GeneMark.hmm: new solutions for gene finding. Nucleic Acids Res 26:11071115. 10.1093/nar/26.4.1107

Morton T, Petricka J, Corcoran DL, Li S, Winter CM, Carda A, Benfey PN, Ohler U, and Megraw M. 2014. Paired-end analysis of transcription start sites in Arabidopsis reveals plantspecific promoter signatures. Plant Cell 26:2746-2760. 10.1105/tpc.114.125617

Mulder N. 2018. Faculty of 1000 evaluation for Identifying noncoding risk variants using diseaserelevant gene regulatory networks. F1000 - Post-publication peer review of the biomedical literature. 10.3410/f.732691059.793543018

Nasiri J, Naghavi M, Rad SN, Yolmeh T, Shirazi M, Naderi R, Nasiri M, and Ahmadi S. 2013. Gene identification programs in bread wheat: a comparison study. Nucleosides Nucleotides Nucleic Acids 32:529-554. 10.1080/15257770.2013.832773

Pritsker M, Liu Y-C, Beer MA, and Tavazoie S. 2004. Whole-genome discovery of transcription factor binding sites by network-level conservation. Genome Res 14:99-108. 10.1101/gr. 1739204

Rye M, Sandve GK, Daub CO, Kawaji H, Carninci P, Forrest ARR, Drabløs F, and consortium F. 2014. Chromatin states reveal functional associations for globally defined transcription start sites in four human cell lines. BMC Genomics 15:120. 10.1186/1471-2164-15-120

Salamov AA, and Solovyev VV. 2000. Ab initio gene finding in Drosophila genomic DNA. Genome Res 10:516-522.

Sandelin A, Carninci P, Lenhard B, Ponjavic J, Hayashizaki Y, and Hume DA. 2007. Mammalian RNA polymerase II core promoters: insights from genome-wide studies. Nat Rev Genet 8:424-436. 10.1038/nrg2026

Sanusi NSNM, Rosli R, Halim MAA, Chan K-L, Nagappan J, Azizi N, Amiruddin N, Tatarinova TV, and Low E-TL. 2018. PalmXplore: oil palm gene database. Database 2018. 10.1093/database/bay095

Shahmuradov IA, and Solovyev VV. 2015. Nsite, NsiteH and NsiteM computer tools for studying transcription regulatory elements: Fig. 1. Bioinformatics 31:3544-3545. 10.1093/bioinformatics/btv404

Shahmuradov IA, Umarov RK, and Solovyev VV. 2017. TSSPlant: a new tool for prediction of plant Pol II promoters. Nucleic Acids Res 45:e65. 10.1093/nar/gkw1353

Sheshadri SA, Nishanth MJ, Harita N, Brindha P, and Bindu S. 2018. Comparative genome based cis-elements analysis in the $5^{\prime}$ upstream and $3^{\prime}$ downstream region of cell wall invertase and Phenylalanine ammonia lyase in Nicotiana benthamiana. Comput Biol Chem 72:181191. 10.1016/j.compbiolchem.2017.11.004

Smale ST, and Baltimore D. 1989. The "initiator" as a transcription control element. Cell 57:103113. $10.1016 / 0092-8674(89) 90176-1$

Solovyev VV, and Shahmuradov IA. 2003. PromH: Promoters identification using orthologous genomic sequences. Nucleic Acids Res 31:3540-3545.

Solovyev VV, Shahmuradov IA, and Salamov AA. 2010. Identification of Promoter Regions and Regulatory Sites. Methods in Molecular Biology, 57-83.

Stanke M, and Morgenstern B. 2005. AUGUSTUS: a web server for gene prediction in eukaryotes that allows user-defined constraints. Nucleic Acids Res 33:W465-467. 10.1093/nar/gki458

Peer] reviewing PDF | (2019:06:38847:1:3:NEW 25 Sep 2019) 
517

518

519

520

521

522

523

524

525

526

527

528

529

530

531

532

533

534

535

536

537

538

539

540

541

542

543

544

545

546

547

548

549

550

551

552

553

554

555

556

557

558

559

Steijger T, Abril JF, Engström PG, Kokocinski F, Consortium R, Hubbard TJ, Guigó R, Harrow J, and Bertone P. 2013. Assessment of transcript reconstruction methods for RNA-seq. Nat Methods 10:1177-1184. 10.1038/nmeth.2714

Suzuki A, Kawano S, Mitsuyama T, Suyama M, Kanai Y, Shirahige K, Sasaki H, Tokunaga K, Tsuchihara K, Sugano S, Nakai K, and Suzuki Y. 2018. DBTSS/DBKERO for integrated analysis of transcriptional regulation. Nucleic Acids Res 46:D229-D238. 10.1093/nar/gkx1001

Tatarinova T, Elhaik E, and Pellegrini M. 2013a. Cross-species analysis of genic GC3 content and DNA methylation patterns. Genome Biol Evol 5:1443-1456. 10.1093/gbe/evt103

Tatarinova T, Kryshchenko A, Triska M, Hassan M, Murphy D, Neely M, and Schumitzky A. 2013b. NPEST: a nonparametric method and a database for transcription start site prediction. Quantitative Biology 1:261-271. 10.1007/s40484-013-0022-2

Tatarinova TV, Chekalin E, Nikolsky Y, Bruskin S, Chebotarov D, McNally KL, and Alexandrov N. 2016. Nucleotide diversity analysis highlights functionally important genomic regions. Sci Rep 6:35730. 10.1038/srep35730

Triska M, Ivliev A, Nikolsky Y, and Tatarinova TV. 2017a. Analysis of cis-Regulatory Elements in Gene Co-expression Networks in Cancer. Methods Mol Biol 1613:291-310. 10.1007/9781-4939-7027-8_11

Triska M, Solovyev V, Baranova A, Kel A, and Tatarinova TV. 2017b. Nucleotide patterns aiding in prediction of eukaryotic promoters. PLoS One 12:e0187243. 10.1371/journal.pone.0187243

Troukhan M, Tatarinova T, Bouck J, Flavell RB, and Alexandrov NN. 2009. Genome-Wide Discovery of cis-Elements in Promoter Sequences Using Gene Expression. OMICS 13:139-151. 10.1089/omi.2008.0034

Umarov RK, and Solovyev VV. 2017. Recognition of prokaryotic and eukaryotic promoters using convolutional deep learning neural networks. PLoS One 12:e0171410. 10.1371/journal.pone.0171410

Weltmeier F, Ehlert A, Mayer CS, Dietrich K, Wang X, Schütze K, Alonso R, Harter K, VicenteCarbajosa J, and Dröge-Laser W. 2006. Combinatorial control of Arabidopsis proline dehydrogenase transcription by specific heterodimerisation of bZIP transcription factors. EMBO J 25:3133-3143. 10.1038/sj.emboj.7601206

Yang T, and Poovaiah BW. 2002. A calmodulin-binding/CGCG box DNA-binding protein family involved in multiple signaling pathways in plants. J Biol Chem 277:45049-45058. 10.1074/jbc.M207941200

Yao H, Guo L, Fu Y, Borsuk LA, Wen T-J, Skibbe DS, Cui X, Scheffler BE, Cao J, Emrich SJ, Ashlock DA, and Schnable PS. 2005. Evaluation of five ab initio gene prediction programs for the discovery of maize genes. Plant Mol Biol 57:445-460. 10.1007/s11103-005-02711

Zhang S-L, Dong-fang LI, Zhang G-S, Wang J-W, and Na NIU. 2008. The Prediction of Rice Gene by Fgenesh. Agric Sci China 7:387-394. 10.1016/s1671-2927(08)60081-4

Zhu Q, Dabi T, and Lamb C. 1995. TATA Box and Initiator Functions in the Accurate Transcription of a Plant Minimal Promoter in vitro. Plant Cell 7:1681. 10.2307/3870029

Peer) reviewing PDF | (2019:06:38847:1:3:NEW 25 Sep 2019) 
Figure 1

TransPrise algorithm. TransPrise algorithm. Inset: CNN architecture that was used in classification/regression model training

CNN architecture was used in classification and regression steps 


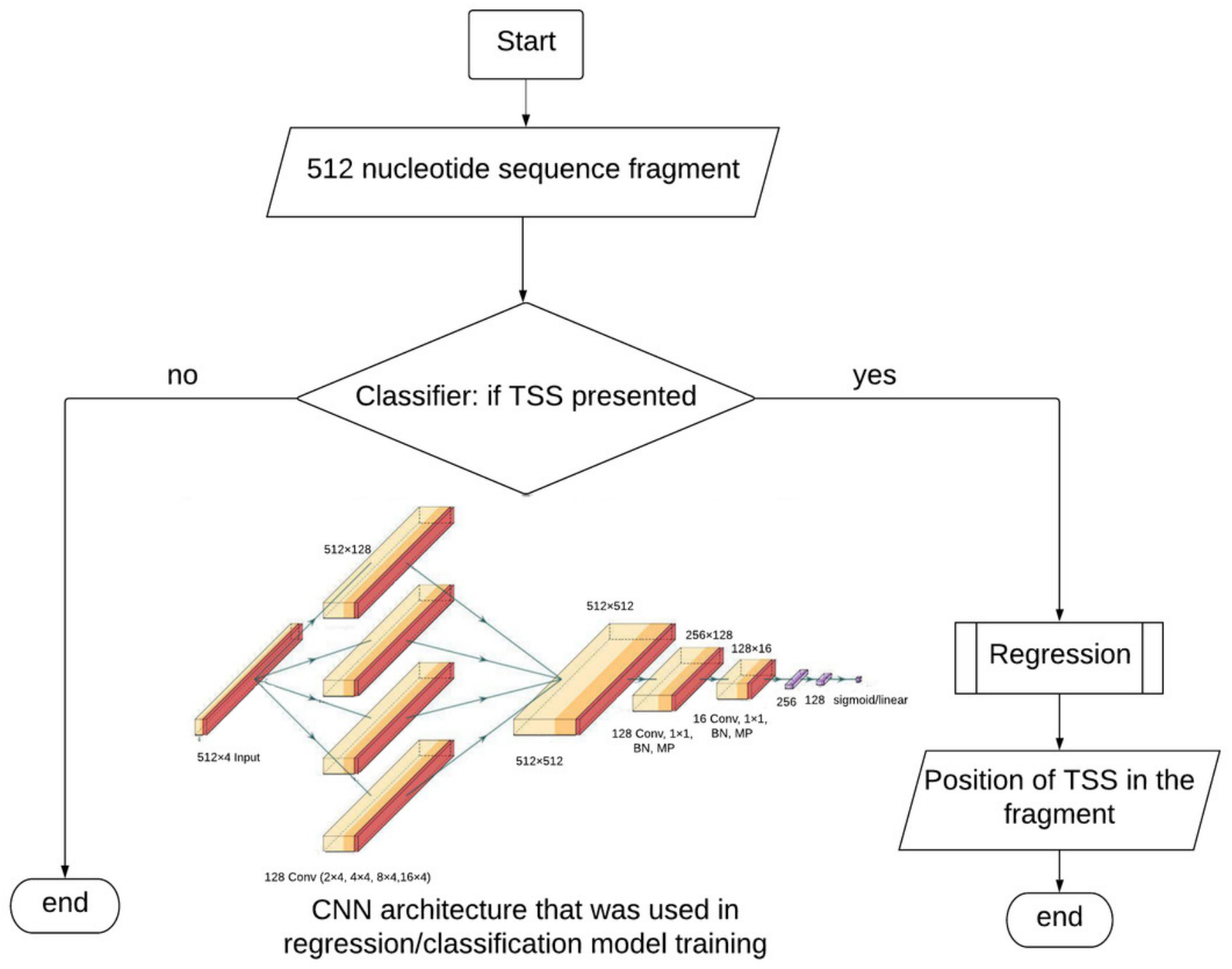


Figure 2

Flowchart of model building and validation.

The figure presents a flowchart of the model building and validation.

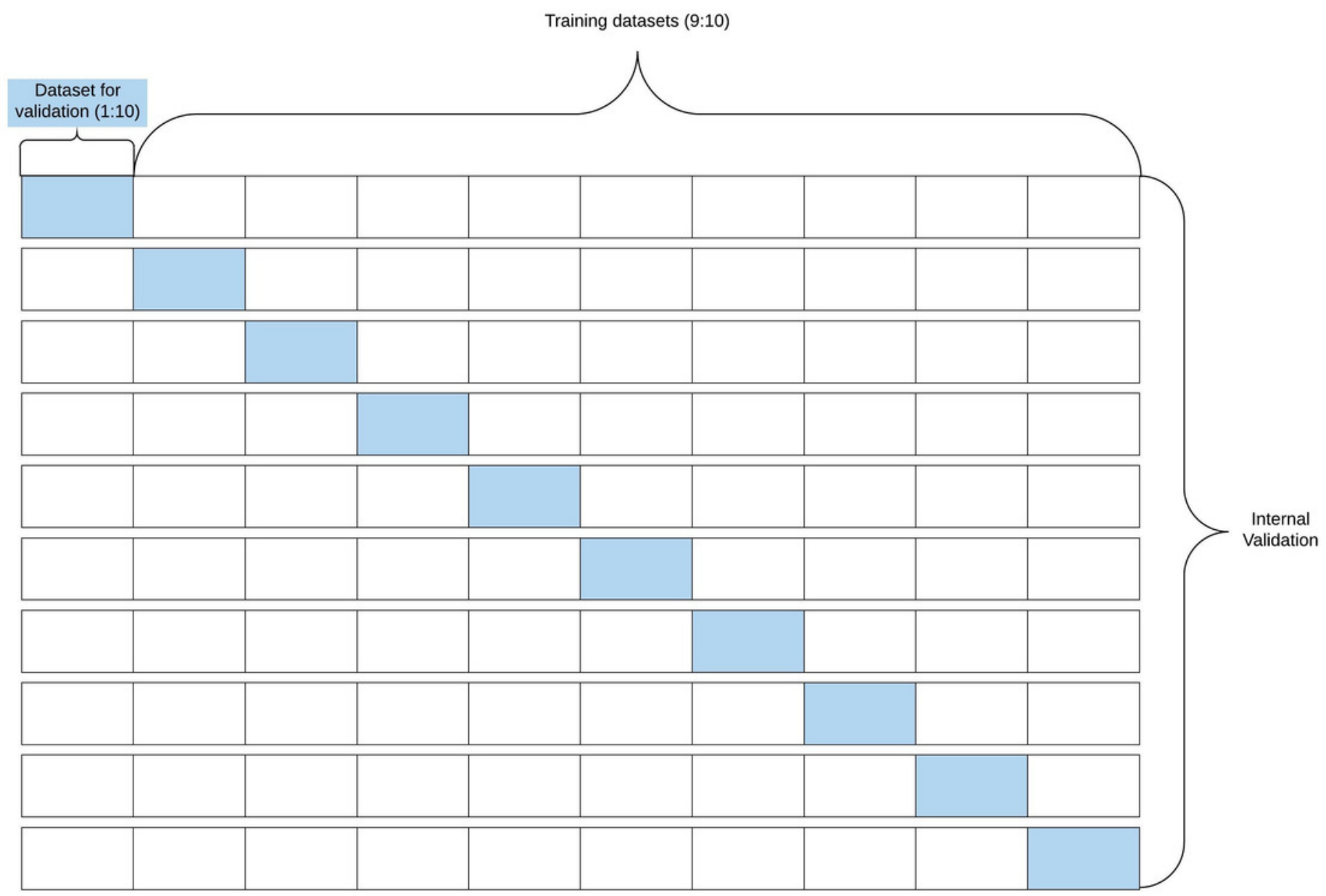

Selecting of the best model 
Figure 3

ROC curve for the training dataset

ROC-curves obtained 10-fold cross-validation procedure of classification model. A: training dataset, $\mathrm{B}$ : testing dataset. Accuracy $=0.88, \mathrm{Se}=0.84, \mathrm{Sp}=0.92, \mathrm{CC}=0.79, \mathrm{AUC}=0.94$ 


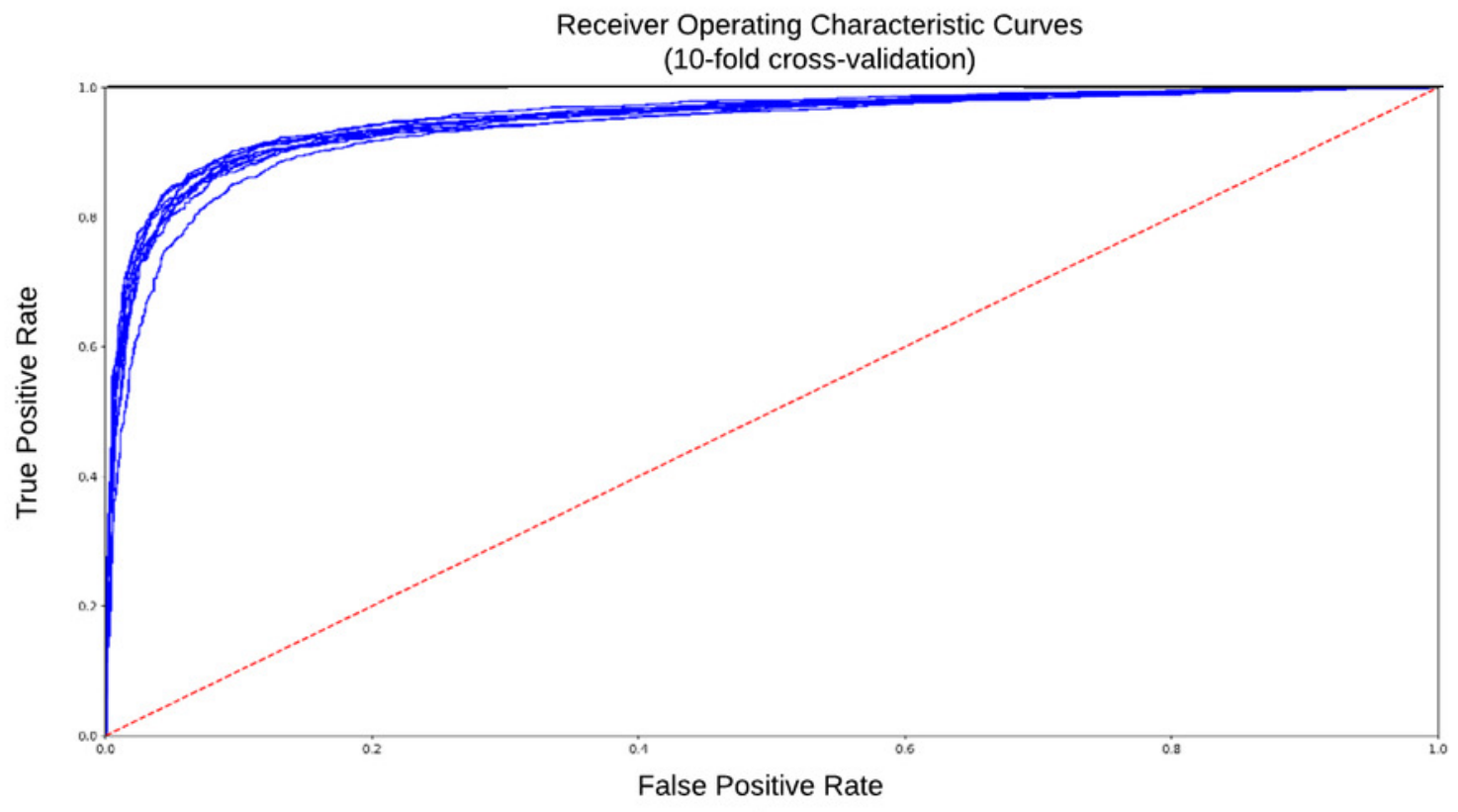

A

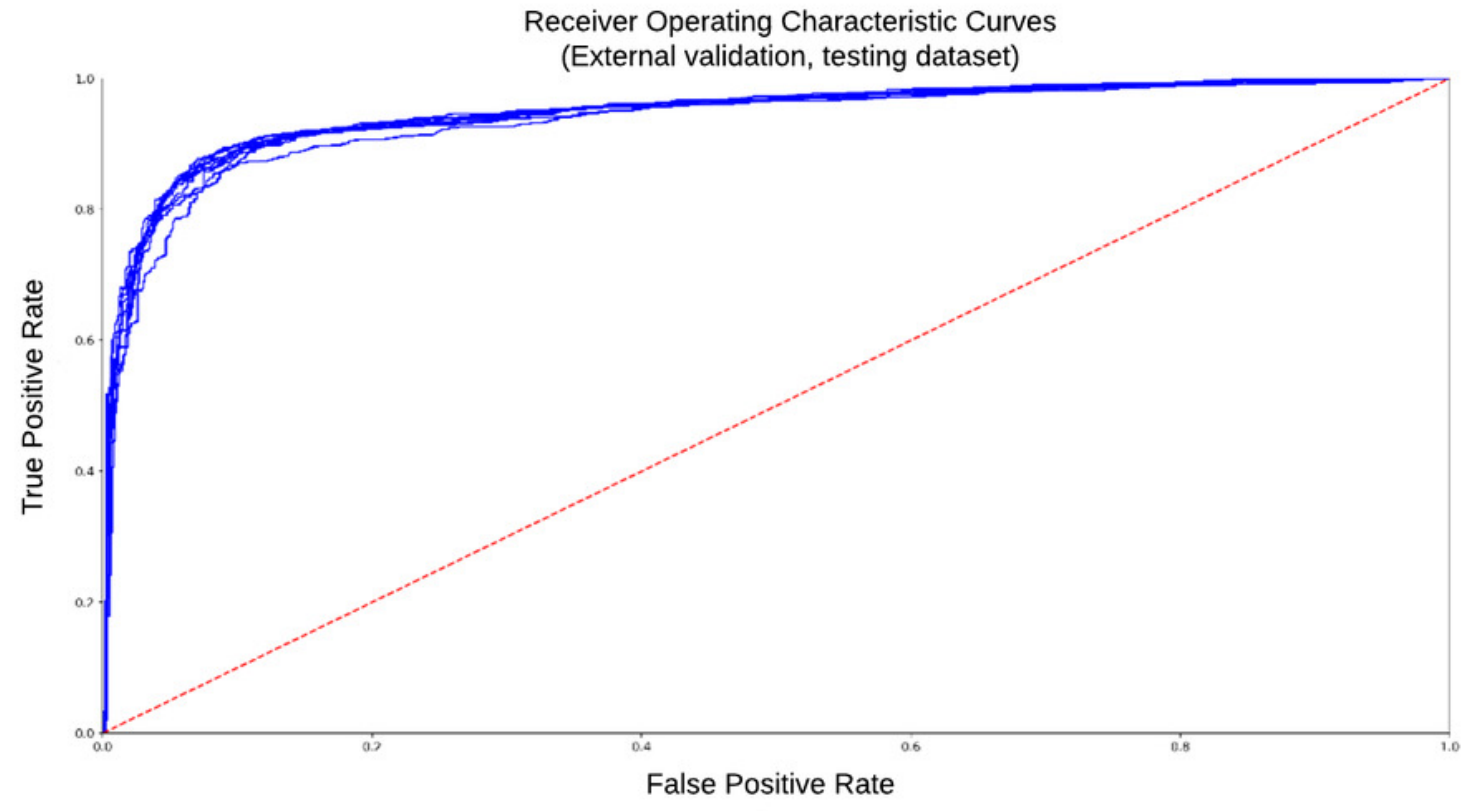

B 


\section{Figure 4}

Error density curves obtained in 10-fold cross-validation of regression models.

The error density curves were obtained in the 10 -fold cross validation procedure for regression models. The mean absolute error (MAE) for regression model was $47.68 \mathrm{nt}$. The testing was performed for entire chromosomes, without filtering for possible TSS containing regions, located upstream of the ATG.

Error densities curves (10-fold cross-validation)

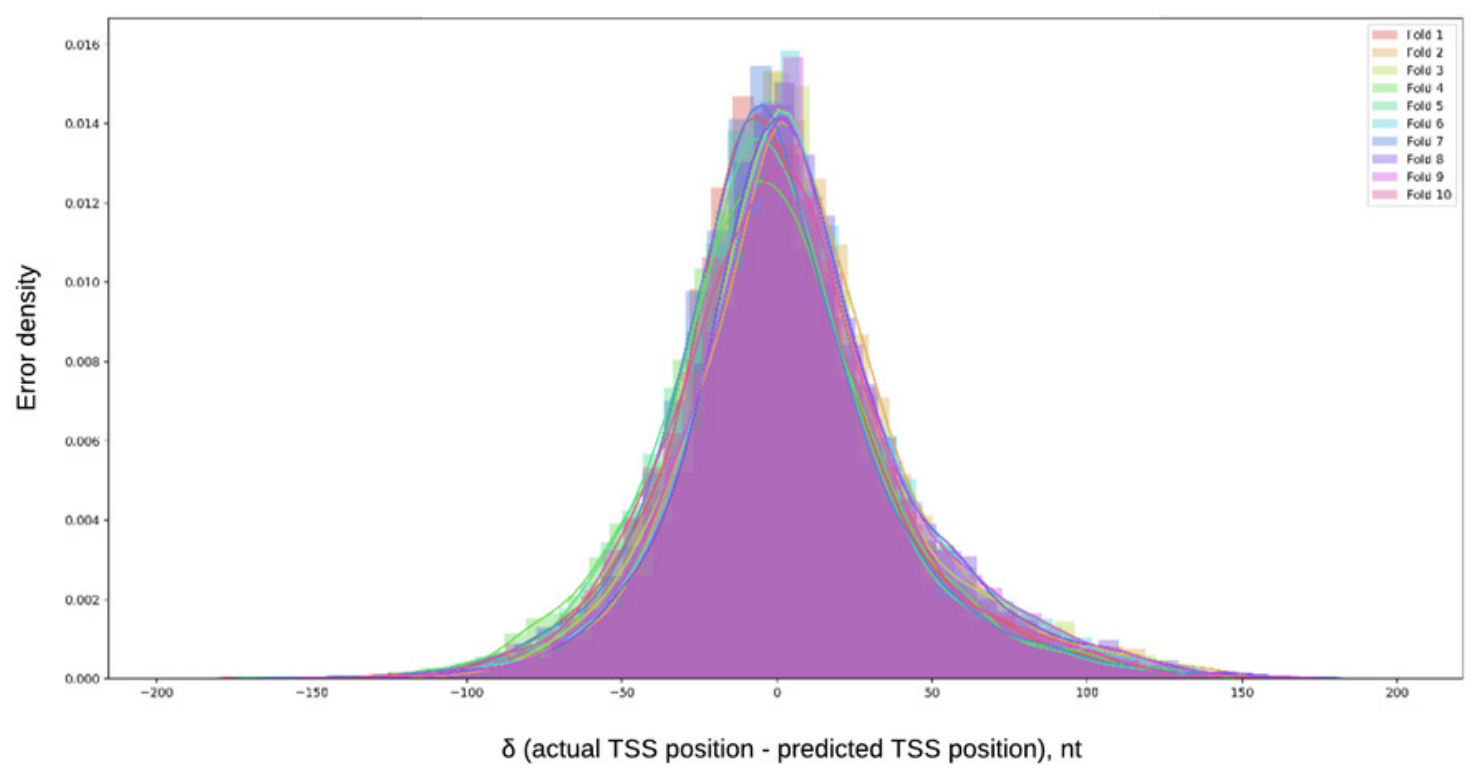




\section{Figure 5}

Comparison between TransPrise and TSSPlant predictions (fraction of sites)

TransPrise predictions tend to be closer to validated TSS than TSSPlant predictions. A:

number of predictions, B: fraction of prediction. 

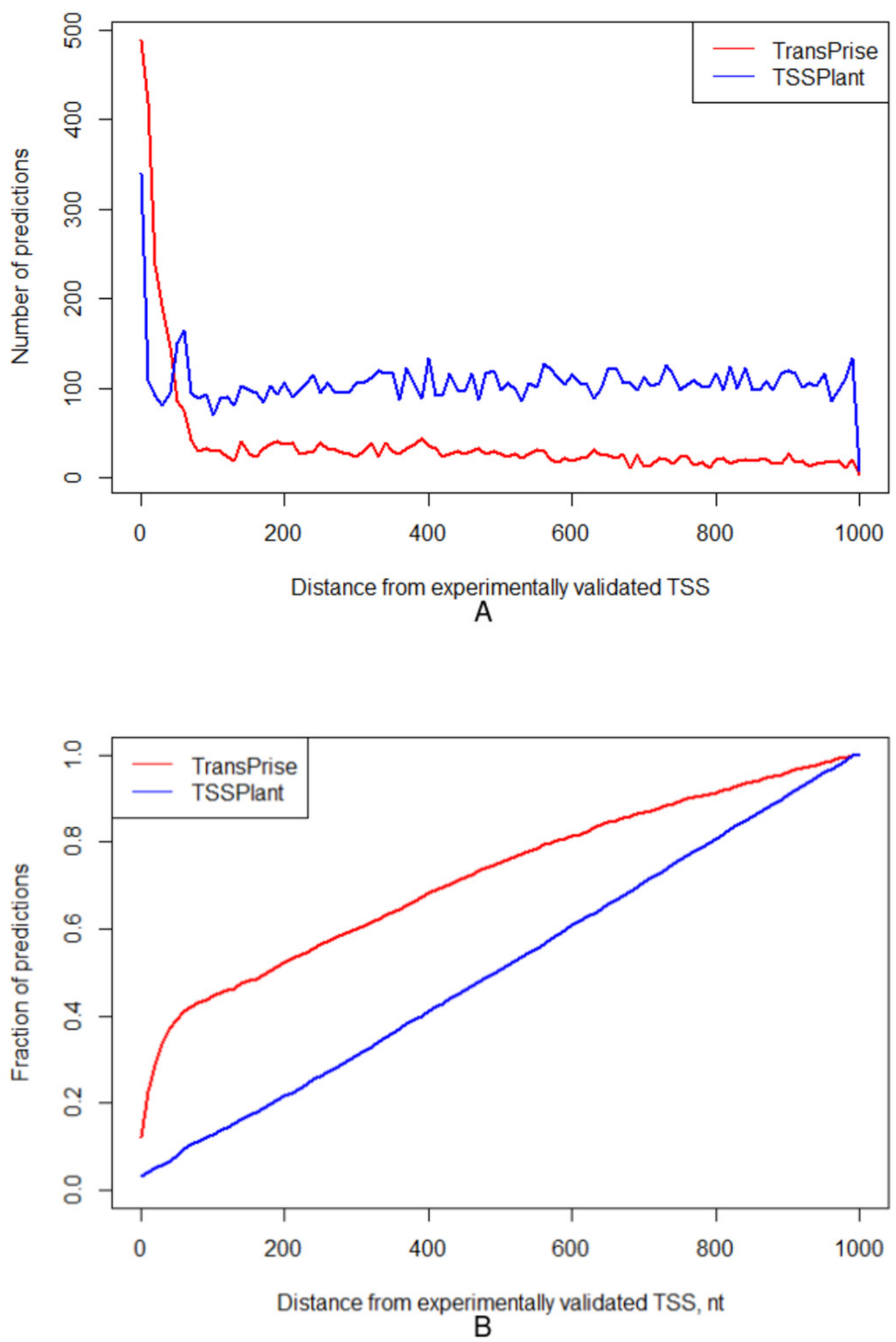
Figure 6

Distribution of CA and TATA motifs in promoter region.

A: Distribution of the CA motif has a peak at TSS (position 0). B: TATA motif is frequently located at position -30 , its density shows a peak at -30 .
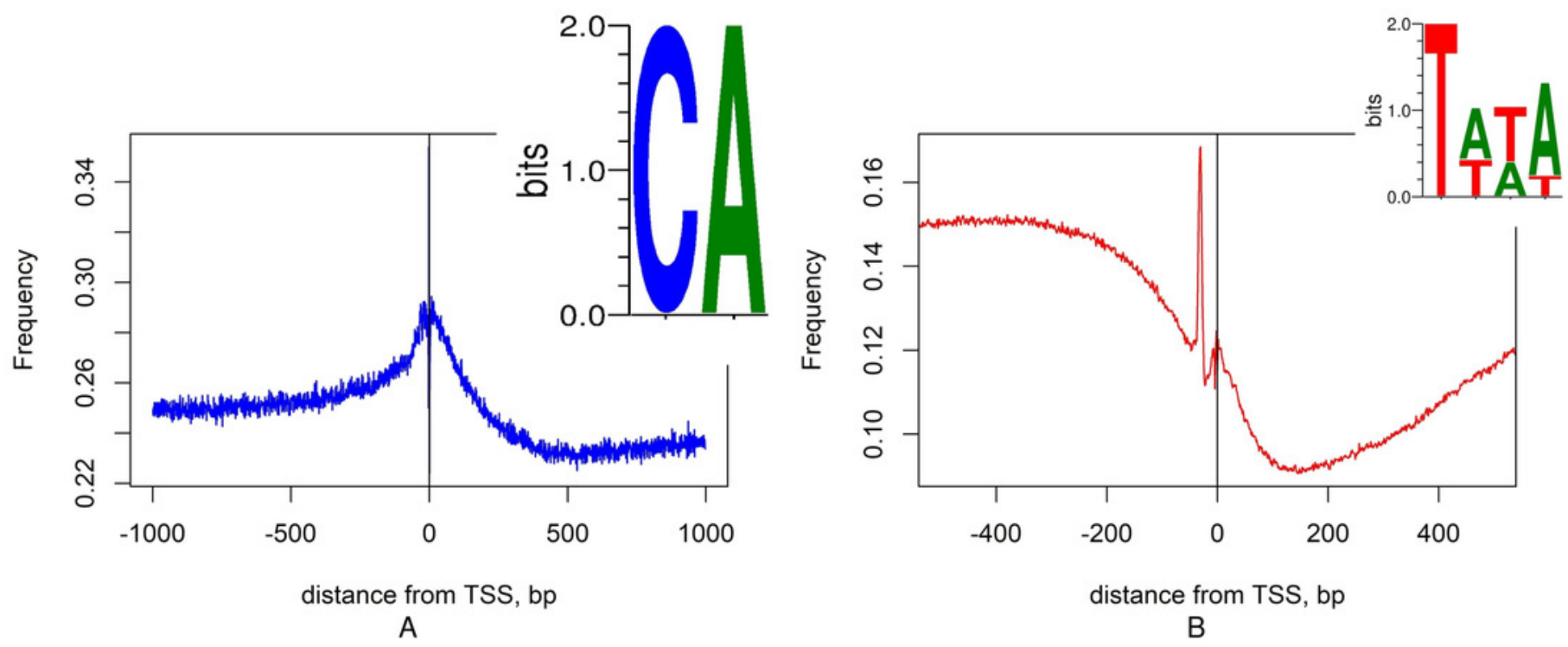


\section{Table $\mathbf{1}$ (on next page)}

Table 1: Comparison of accuracy metrics of TransPrise and TSSPlant classification models

TransPrise offers higher accuracy compared to the TSSPlant 
1 Table 1: Comparison of accuracy metrics of TransPrise and TSSPlant classification models

\begin{tabular}{|l|l|l|l|l|l|}
\hline $\begin{array}{l}\text { Classification } \\
\text { Model }\end{array}$ & MCC & Accuracy & Sensitivity & Specificity & AUC \\
\hline TSSPlant & 0.310 & 0.603 & 0.976 & 0.231 & 0.603 \\
\hline TransPrise & 0.791 & 0.895 & 0.872 & 0.919 & 0.952 \\
\hline
\end{tabular}

2

3 